\title{
Platelet Derived Growth Factor BB: A "Must-have" Therapeutic Target "Redivivus" in Ovarian Cancer
}

\author{
ANCA MARIA CIMPEAN ${ }^{1}$, IONUT MARCEL COBEC ${ }^{2}$, RALUCA AMALIA CEAUȘU ${ }^{1}$, \\ ROXANA POPESCU ${ }^{3}$, ANCA TUDOR $^{4}$ and MARIUS RAICA ${ }^{1}$ \\ Departments of ${ }^{1}$ Microscopic Morphology/Histology, Angiogenesis Research Center, \\ ${ }^{3}$ Microscopic Morphology/ Cell and Molecular Biology, ${ }^{4}$ Medical Informatics and Biostatistics, \\ Victor Babes University of Medicine and Pharmacy, Timisoara, Romania; \\ ${ }^{2}$ Department of Obstetrics and Gynecology, Diakonie Klinikum, \\ Academic Hospital of the Heidelberg University, Schwäbisch Hall, Germany
}

\begin{abstract}
Background: We aimed to validate PDGF-BB protein expression by RNAscope, a sensitive method for $P D G F-B B$ mRNA evaluation on paraffin embedded (FFPE) specimens of ovarian tumors. Materials and Methods: Seventy-five FFPE ovarian cancer biopsies were assessed by immunohistochemistry followed by PDGF-BB mRNA RNAscope validation. Results and Conclusion: Dual PDGF$B B$ expression in tumor and stromal cells have been observed, being highly suggestive for PDGF-BB mediated stromal-tumor cells reciprocal interaction in ovarian cancer $(p=0.008)$. It seems that the nuclear expression of the $P D G F-B B$ represents a negative prognostic factor in ovarian tumors. Being a controversial issue in the literature, PDGF$B B$ nuclear expression detected by immunohistochemistry was validated by RNAscope in situ hybridization. More than 65\% of cases had PDGF-BB mRNA amplification, confirming immunohistochemical results. We herein validated $P D G F-B B$ as a potential therapeutic and prognostic tool of ovarian cancer aggressiveness.
\end{abstract}

Platelet derived growth factors (PDGFs) family includes several members involved in cell growth and proliferation control. They are also known to be responsible for certain steps of new blood vessel development (angiogenesis), mainly in vessel growth and maturation by recruiting perivascular cells. Excessive angiogenesis is mainly a feature of malignant

Correspondence to: Anca Maria Cimpean, MD, Ph.D., Professor of Histology, Department of Microscopic Morphology/Histology, Angiogenesis Research Center Timisoara, Pta Eftimie Murgu 2, 300041 Timisoara, Romania. Tel: +40 720060955, e-mail: ancacimpean1972@yahoo.com

Key Words: PDGF-BB, RNAscope, therapy, ovarian carcinoma. development, but also represents an important component for other pathologic conditions, such as diabetes or arthritis. PDGFs family members are presented as dimeric glycoproteins with three isoforms $\mathrm{AA}, \mathrm{BB}, \mathrm{AB}$. They also represent a strong mitogen for cells with mesenchymal origin, such as fibroblasts, smooth muscle cells or glial cells $(1,2)$. PDGF-BB isoform is secreted by both tumor and endothelial cells (3). Based on the observation that PDGF-BB is expressed in several and various tumor cells and neovessels, many authors studied it as a potential therapeutic target with a dual role regarding concomitant inhibition of tumor growth and vessels development. Several anti-PDGFs therapies are currently being tested with a large applicability, especially in ophthalmology. As such an example, a PDGF-BB inhibitor called Fovista ${ }^{\circledR}$ (Ophthotech, New York, NY, USA) may improve bevacizumab effects and it is used on a large scale as inhibitor of the angiogenesis developed during macular degeneration (4).

The interrelation and mutual influence between PDGF-BB and VEGF was observed for ovarian tumors too and was recently reported by Matei and coworkers (5). The authors observed that PDGF-BB induced VEGF synthesis in ovarian carcinomas and PDGF receptor-beta (PDGFR $\beta$ ) inhibition by imatinib produced a dramatic decrease of serum VEGF in ovarian carcinomas. $\mathrm{Lu}$ and his team underlined the increased efficiency of the anti-PDGFB therapy on an experimental model of ovarian carcinomas developed from human tumor cells through dual, concomitant target of the endothelial and perivascular cells by a combination of bevacizumab and an anti-PDGF aptamer (6).

Recent data strongly sustain a combined anti-VEGF-antiPDGF therapy in ovarian cancer (7). Despite of preliminary results of the experimental in vitro and in vivo studies that sustain such combination, data on PDGF-BB expression of human ovarian cancer specimens are extremely rare, being reported in no more than fifteen articles in PubMed. 
Immunohistochemistry represents the first routine method assessed to certify the presence of growth factors secreted by tumor cells at the protein level only. Sometimes, falsepositive results may be obtained due to cross-reaction with any protein from the tissue. Thus, an accurate assessment of any marker must be completed with growth factors gene amplification by using different methods. RNAscope is an in situ hybridization method which identifies mRNA gene amplification of any marker previously characterized by immunohistochemistry on formalin-fixed paraffin-embedded specimens (FFPE). It allows the detection of single RNA molecules in single cells. It has the advantage to be performed on FFPE sections and it allows correlations between $P D G F-B B$ mRNA amplification and histopathological features of ovarian tumors and, moreover to quantify heterogeneity of PDGF-BB distribution inside ovarian cancer. Assessment of presence and heterogeneity of $P D G F-B B$ mRNA in ovarian cancer may be mandatory in the future for an accurate quantification of the expression followed by a personalized targeted therapy against PDGFBB. RNAscope technology is already used in clinical practice for other cancer types but not yet for ovarian cancer.

Based on the facts described above, we aimed to evaluate ovarian cancer PDGF-BB expression by immunohistochemistry followed by its validation using RNA scope, a sensitive and reliable method that gives us the opportunity to assess PDGF-BB mRNA on formalin-fixed, paraffin embedded (FFPE) human specimens. This multimodal approach may be useful as an additional tool for a more accurate evaluation of ovarian cancer with a direct impact on PDGF-BB validation as a therapeutic target for specific therapies, already approved in other cancer types.

\section{Materials and Methods}

Our study included seventy-five archival paraffin-embedded specimens of ovarian cancer that were selected by two experienced pathologists.

Specimen re-evaluation and selection. Both pathologists reviewed histopathological diagnosis and selected cases suitable for immunohistochemistry and RNAscope methods. They excluded 8 cases from those initially selected, based on an improper fixation (negative immunohistochemical reaction for vimentin, clone V9, used as marker highlighting proper fixation). Three micrometers (for immunohistochemistry) and five micrometers (for RNAscope in situ hybridization) sections were performed from each remained 67 FFPE specimens.

Immunohistochemistry. Immunohistochemistry was performed in a fully automated manner by using BOND MAX Autostainer (Leica Microsystems, Newcastle, UK) and a compatible standardized kit Bond Polymer Refine Detection System Brown followed the incubation with PDGF-BB primary antibody (rabbit, polyclonal, dilution 1:200, Thermo Fisher Scientific Anatomic Pathology, LabVision, Cheshire, UK). Nuclear and cytoplasmic expression was considered proper for interpretation regarding positive cell density and intensity.
RNAscope in situ hybridisation method. By this technique, celular RNA content was assessed as a single-molecule visualisation in individual cells on FFPE specimens through use of a novel probe design strategy and a hybridization-based signal amplification system to simultaneously amplify signals and suppress background. HRP-conjugated double $\mathrm{Z}$ probes for PDGF-BB, complementary for the target RNA were designed by Advanced Cell Diagnostics (Hayward, CA, USA). We used RNAscope 2.0 High Definition Kit (Advanced Cell Diagnostics, Hayward, CA, USA) for signal amplification followed by visualisation of amplified signals with diaminobenzidine as chromogen.

Briefly, to prepare FFPE specimens for RNAscope procedure, the tissues were fixed and permeabilized to allow for target probe access. Two-hours hybridisation step performed at $40^{\circ} \mathrm{C}$ was followed by multistep signal amplification done by applying reagents followed by RNA visualisation as brown dotted spots with diaminobenzidine. The detection sensitivity of the RNAscope method was proven by using positive (POLR2A) and negative (probes against the bacterial gene dapB) controls assessed by using the same protocol as for pterygium specimens. Interpretation of $P D G F-B B$ mRNA amplification was manually performed in a semi-quantitative manner following scoring guidelines provided by the manufacturer. The presence of $P D G F-B B$ mRNA amplification was scored into five grades as 0 (no staining or less than 1 dot to every 10 cells, $40 \times$ magnification), 1 (1-3 dots/cell visible at 20-40X magnification), 2 (4-10 dots/cell, very few dot clusters visible at 20-40× magnification), 3 (>10 dots/cell, less than $10 \%$ positive cells have dot clusters visible at $20 \times$ magnification) and 4 (>10 dots/cell, more than $10 \%$ positive cells have dot clusters visible at $20 \times$ magnification).

Image aquisition and statistical analysis. Microscopic evaluation was performed by using AxioCam 506 Color Research Microscope (Zeiss, Jena, Germany) equipped with a software specific for automated transfer of the images from the camera to the computer. SPSS software version 19 was used to perform statistical analysis of data derived from histopathological, immunohistochemical and molecular assessement of PDGF-BB in ovarian cancer specimens. Statistical correlations were considered significant if their value were less than $0.05(p \leq 0.05)$.

\section{Results}

Normal ovary adjacent to tumor tissue expressed PDGF-BB in stromal cells of ovarian cortex and medulla (more intense in those from cortex compared with stromal cells from the medulla). Capillaries inside cortical stroma were highly positive for PDGF-BB (expressed by endothelial cells). Intensity of immunohistochemical reaction for PDGF-BB constantly decreased from albuginea to the deeper part of the cortex, being weak or absent inside and around albicans bodies (Figure 1a and $\mathrm{b}$ ).

From the total number of cases, $22.4 \%$ were benign tumors (dermoid cysts, thecomas, cystadenomas) while the rest of $79.6 \%$ of cases were serous cystadenocarcinomas (65.7\%) and endometrioid carcinomas (11.9\%).

A percent of $62.5 \%$ out of total number of benign tumors did not express PDGF-BB, nor in stroma neither in tumor areas. Compared to benign lesions, the malignant ones 
Table I. The procentual expression of the PDGF-BB pozitive cases, dependent by intensity of immunohistochemical expression in tumor cells.

\begin{tabular}{lcr}
\hline$P D G F-B B$ score in tumor cells & Nr. of cases & $\%$ \\
\hline 0 & 8 & 11.9 \\
1 & 5 & 7.5 \\
2 & 10 & 14.9 \\
3 & 44 & 65.7 \\
Total & 67 & 100.0 \\
\hline
\end{tabular}

Table II. Distribution of PDGF-BB expression patterns in ovarian tumors inlcuded in the study.

\begin{tabular}{lcr}
\hline$P D G F-B B$ stromal & Nr. of cases & $\%$ \\
\hline 0 & 21 & 31.3 \\
1 & 10 & 14.9 \\
2 & 10 & 14.9 \\
3 & 26 & 38.8 \\
Total & 67 & 100.0 \\
\hline
\end{tabular}

Table III. Case distribution according with immunohistochemical score given for stromal compartment for PDGF-BB expression in ovarian tumors.

\begin{tabular}{lcc}
\hline$P D G F-B B$ immunohistochemical pattern & Nr. of cases & $\%$ \\
\hline 0 & 7 & 10.4 \\
Cytoplasmic & 11 & 16.4 \\
Nuclear & 24 & 35.8 \\
Combined & 25 & 37.3 \\
Total & 67 & 100.0 \\
\hline
\end{tabular}

presented an intense PDGF-BB expression with a heterogeneous pattern found both in tumor and stromal cells. The most common pattern was a combination between nuclear and cytoplasmic expression.

About $88 \%$ out of total number of ovarian cancers were PDGF-BB-positive by immunohistochemistry. PDGF-BB was observed both in tumor and stromal cells with a combined nuclear and cytoplasmic expression dependent by tumor type. For serous cystadenocarcinoma, papillary type, PDGF-BB expression combined both nuclear and cytoplasmic localization (Figure 1c) stromal expression being still present (Figure 1d). Small blood vessels of tumor stroma had a constant PDGF-BB expression at the endothelial level (Figure 1f). Compact serous adenocarcinomas tumor cells had weak cytoplasmic and intense nuclear PDGF-BB expression (Figure 1e), while endometrioid type was characterized by cytoplasmic restricted PDGF- BB expression (Figure $1 \mathrm{~g}$ and $\mathrm{h}$ ).
Table IV. Interrelation between tumor stromal PDGF-BB expression.

\begin{tabular}{|c|c|c|c|c|c|}
\hline \multirow{2}{*}{$\begin{array}{l}P D G F-B B \text { in } \\
\text { tumor cells }\end{array}$} & \multicolumn{4}{|c|}{ PDGFB stromal cells } & \multirow[t]{2}{*}{ Total } \\
\hline & 0 & 1 & 2 & 3 & \\
\hline \multirow[t]{2}{*}{0} & 7 & 0 & 0 & 1 & 8 \\
\hline & $87.5 \%$ & $0 \%$ & $0 \%$ & $12.5 \%$ & $100.0 \%$ \\
\hline \multirow[t]{2}{*}{1} & 3 & 1 & 1 & 0 & 5 \\
\hline & $60.0 \%$ & $20.0 \%$ & $20.0 \%$ & $0 \%$ & $100.0 \%$ \\
\hline \multirow[t]{2}{*}{2} & 4 & 2 & 2 & 2 & 10 \\
\hline & $40.0 \%$ & $20.0 \%$ & $20.0 \%$ & $20.0 \%$ & $100.0 \%$ \\
\hline \multirow[t]{2}{*}{3} & 7 & 7 & 7 & 23 & 44 \\
\hline & $15.9 \%$ & $15.9 \%$ & $15.9 \%$ & $52.3 \%$ & $100.0 \%$ \\
\hline
\end{tabular}

For what PDGF-BB expression is concerned in tumor cells, the majority of cases presented intense and moderate expression, and only in approximately $7.5 \%$ of the cases we met a reduced expression noted +1 or being negative. For the tumor stroma prevailed the weak and moderate expression, tumor-stroma border being characterized by a high amount of PDGF-BB-positive cells with fibroblast- or myofibroblastlike morphology grouped around the tumor tissue. Data regarding expression intensity in tumor cells and evidences of expression patterns (nuclear and/or cytoplasmic) are summarized in Tables I and II.

Moreover, we observed the PDGF-BB to the stromal level, this being noted from 0 to +3 . We found that $38.8 \%$ of cases presented a strong expression of the stromal PDGF-BB noted as $+3,14.9 \%$ had a moderate stromal PDGF-BB expression noted +2 , and a similar percentage of $14.9 \%$ stromal PDGF$\mathrm{BB}$ expression was noted as +1 . Thirty one percent of cases do not express PDGF-BB stromal (Table III).

A strong significant correlation was obtained between serous cystadenocarcinoma type and combined, nuclear and cytoplasmic pattern of PDGF-BB immunohistochemical expression $(p<0.001)$.

On the other hand, we followed the intensity of the PDGF-BB expression in tumor cells compared to the intensity of stromal PDGF-BB expression in ovarian tumors included in the study. We observed a correlation between the PDGF-BB expression in tumor and stromal cells and this correlation was proved to be statistically significant $(p=0.008)$ as shown in Table IV.

Our immunohistochemical results required validation by more sensitive methods and thus, RNAscope in situ hybridisation was chosen.

RNAscope method revealed $P D G F-B B$ mRNA genomic amplification in $63.7 \%$ of the cases. About $28.5 \%$ had $P D G F-B B$ mRNA amplification restricted to stromal cells, while, the rest of the cases showed this amplification in both tumor and stromal components. PDGF-BB-negative cases by 

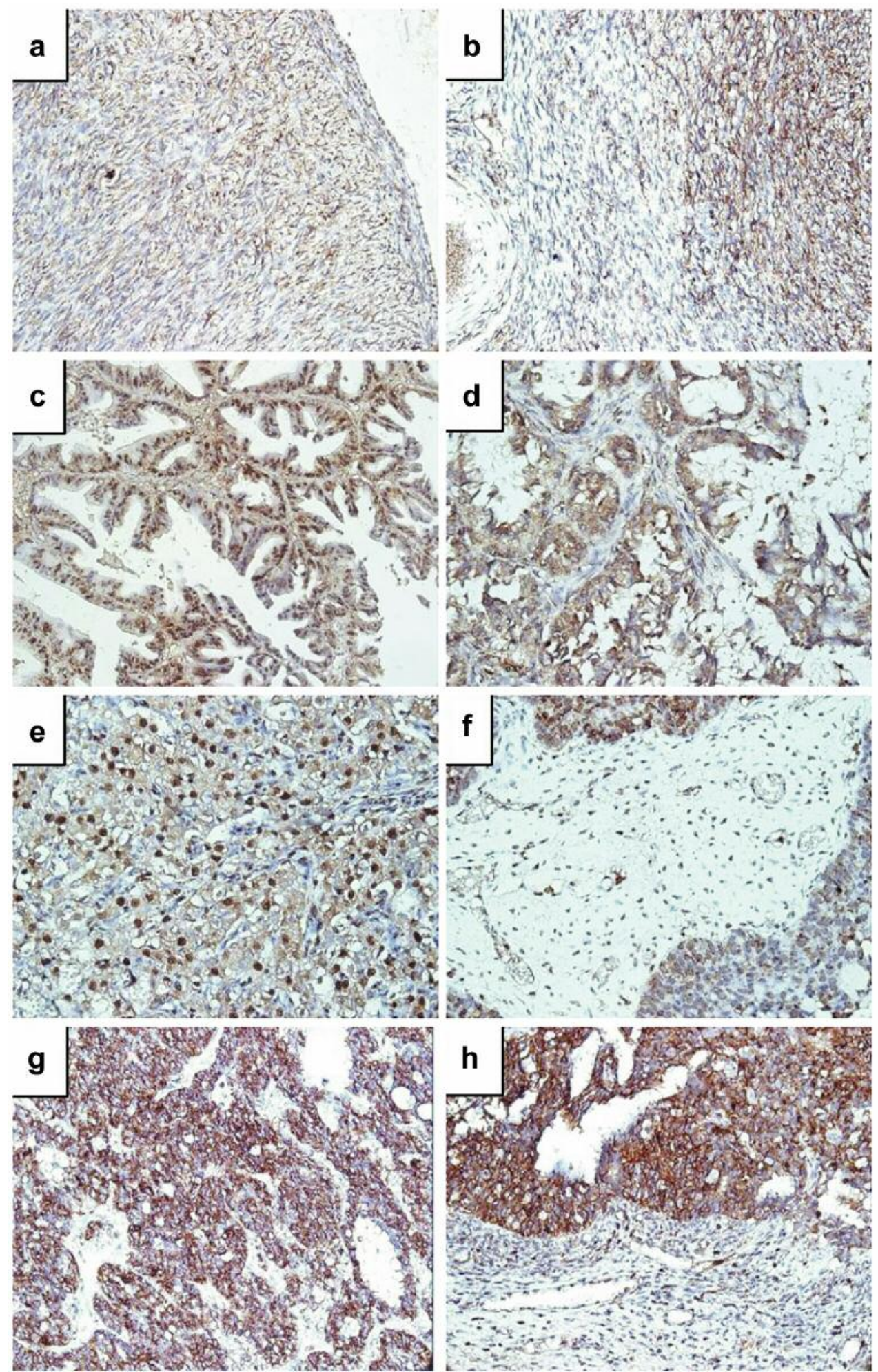

Figure 1. Immunohistochemical PDGF-BB expression in ovarian cancer. Normal ovarian tissue adjacent to tumor tissue expressed PDGF-BB in stromal cells with a higher intensity and cell density in the ovarian cortex (a) compared to medulla (b). In tumor tissue, three expression patterns were identified : nuclear $(c)$, cytoplasmic $(d)$ and combined (e). Tumor stroma fibroblasts and stromal small blood vessels were also positive for $P D G F-B B$, as assessed by immunohistochemistry (f). Endometrioid-type adenocarcinoma was characterized by immunohistochemical expression of $P D G F-B B$ restricted to the cytoplasm of tumor cells $(g, h)$. 


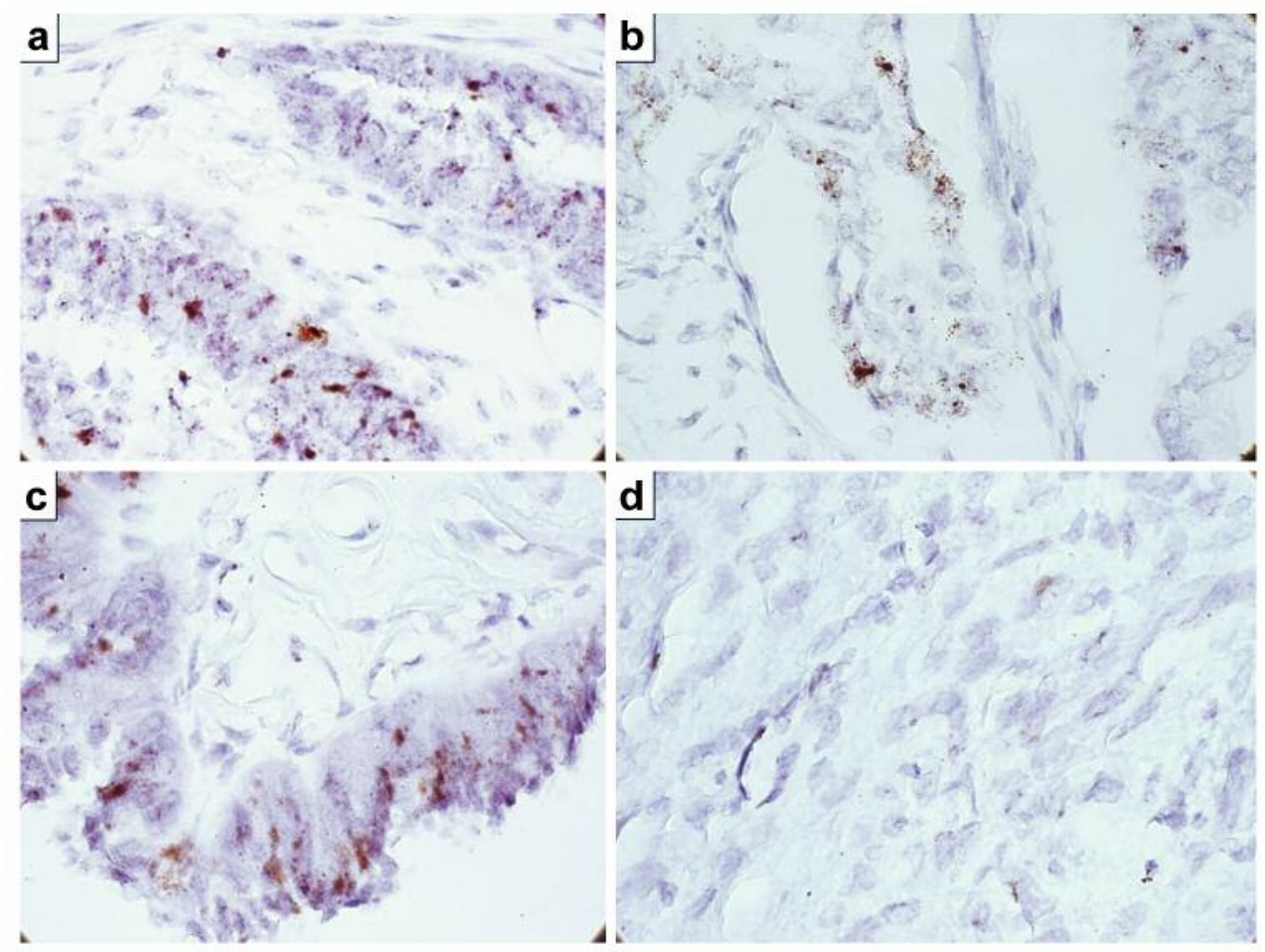

Figure 2. RNAscope in situ hybridization revealed PDGF-BB mRNA amplification with different intensities depending on tumor type. High heterogeneity of PDGF-BB mRNA amplification was recorded inside the same tumor, amplification intensity with score 4 charactrized by clusters of amplification being predominant $(a, c)$. A lower score characterized by single dots dispersed at nuclear and/or cytoplasmic levels confirmed dual immunohistochemical expression previously found $(b)$. Low PDGF-BB mRNA amplification was also present in tumor stroma fibroblasts and endothelial cells from tumor blood vessels $(d)$.

immunohistochemistry were also confirmed as being negative by in situ hybridization. The RNAscope score noted as 4 (clusters and dotted signals) characterized cases with combined nuclear and cytoplasmic immunohistochemical expression with predominant dots distribution and predominantly at the nuclear level. This aspect was observed in the papillary-type tumors (Figure 2). A high $P D G F-B B$ mRNA distribution heterogeneity was proven by RNAscope, areas of PDGF-BB tumor expressing cells being mixed with tumor cells areas lacking $P D G F-B B$ mRNA amplification. Also, a higher $P D G F-B B$ mRNA amplification was counted in stromal areas rich in miofibroblasts compared with tumor stroma where miofibrobalsts are poorly represented.

\section{Discussion}

Ovarian cancer remains a major problem in women's health, because of its aggressiveness and lack of therapeutic efficiency leading to poor prognosis and short survival. Multimodal therapeutic approaches represent the current option but there still remains a great challenge in ovarian cancer treatment. Thus, new drug validation for ovarian cancer with a double action on both malignant cells and newlyformed tumor blood vessels may be a promising alternative to current, low-efficiency therapies $(8,9)$. Growth factor inhibitors are, currently, under continuous improvement and thus, nowadays the only FDA approved therapy for ovarian cancer is that with bevacizumab $(10,11)$. But the partial response of ovarian carcinomas to bevacizumab associated to conventional therapy impose the re-classification of the ovarian cancer through defining some new molecular classes with a strong prognostic and therapeutic impact (12). Recently, Olaparib-associated therapy has been reported to increase long-term survival and improve prognosis, especially for patients which BRCA mutations (13).

Other targeted therapies such as pazopanib, cedirinib, nitedanib, sunitinib, sorafenib or imatinib (14-18) were tested in ovarian cancer, but until now they have not received FDA approval for use in current medical practice. PDGF$\mathrm{BB} / \mathrm{PDGFR} \beta$ axis is less studied in ovarian cancer, with only 14 studies being published on PubMed. Most of them used the plasmatic levels of PDGF-BB for the ovarian cancer patient stratification. Our study pointed-out PDGF-BB expression in tumor and tumor stroma cells, also. 
The percent of ovarian cancer PDGF-BB-positive cases reported by us is similar to those reported in the literature till now (19). The PDGF-BB overexpression in ovarian tumors was reported for the first time by Kacinski and colab. in 1989 (20) and then, several studies (most of them, in vitro studies) were developed (21) but the clinical use of the antiPDGF therapies did not developed significantly.

Our study points-out the importance of in situ hybridization RNAscope method for an accurate PDGF-BB assessment on ovarian cancer specimens. Combining immunohistochemistry and mRNA detection on FFPE we are able to characterize not only PDGF-BB expression but also, we can stratify ovarian cancer patients regarding their response to anti PDGF-BB therapeutic agents. Herein we attempted a complete approach of PDGF-BB evaluation in ovarian cancer. Our data on the identification of $P D G F-B B$ mRNA amplification in more than $60 \%$ of cases are overlapped on similar data found in the literature earlier (22). Previous studies presented images providing us data about PDGF-BB expression pattern as being predominantly cytoplasmic. We herein described three patterns of PDGF-BB expression (nuclear, cytoplasmic or combined) in ovarian cancer.

The nuclear PDGF-BB expression is rarely reported in the literature and it seems to be regulated through the c-sis system, as it has been intensely discussed in metastatic and non-metastatic renal carcinomas $(23,24)$. The c-sis/PDGF axis has been reported to be responsible for the PDGF autocrine effect on tumor cells as it is presented in the literature through several in vitro and in vivo studies (25). This interrelation is hormone dependent and this aspect was not studied in the ovarian tumors, yet (26).

PDGF-BB expression in ovarian tumor stroma is completely neglected. Tumor stromal fibroblasts and myofibroblasts produce PDGF-BB (27). The tumor stroma is considered to be a "malignant" milieu and it controls tumor cells behaviour, just like it is already certified in breast cancer (28). In 2006, Lederle and coworkers proved that PDGF-BB is intensely expressed in tumor fibroblasts and its overexpression stimulate tumor cells proliferation, initially through a transitory growth of VEGFA which act in a paracrine manner and favour tumor progression (29).

Our findings regarding a simultaneously expression of PDGF-BB in tumor and stromal cells from ovarian cancer are in concordance with data presented above. One important issue that might be extensively studied in the future remains PDGF-BB nuclear expression (confirmed by in situ hybridization in our study). This may be responsible for the high aggressiveness and rapid progression, a well-known and hard-to-manage aspect in ovarian cancer. A peculiarity of the present study may consist by the observation of PDGF-BB nuclear expression in the fibroblasts cells of tumor stroma, suggesting the mutual interrelation between them and thus their use as target for anti PDGF therapies. Targeting both stromal and tumor cells represents the gold standard for future oncologic therapeutic options.

\section{Conclusion}

PDGF-BB is expressed not only in tumor cells but also in stromal cells from the tumor stroma of ovarian carcinomas. The expression overlapping in stroma and tumor cells sustain the influence of the stroma over the epithelial cell proliferation in ovarian tumors. Nuclear expression of PDGF-BB may be a negative prognostic factor in ovarian tumors, but this aspect needs further validation. PDGF-BB imunohistochemsitry requires at least one method of molecular validation, at least an in situ hybridization confirmation. Our results sustain the use of anti-PDGF-BB therapy as associated targeted therapy to conventional chemotherapy.

\section{Acknowledgements}

This work was supported by internal funds provided by Victor Babes University of Medicine and Pharmacy Timisoara, Romania.

\section{References}

1 Hannink $M$ and Donoghue DJ: Structure and function of platelet-derived growth factor (PDGF) and related proteins. Biochim Biophys Acta 989: 1-10, 1989.

2 Heldin $\mathrm{CH}$ : Structural and functional studies on platelet-derived growth factor. EMBO J 11: 4251-4259, 1992.

3 Abramsson A, Lindblom P and Betsholtz C: Endothelial and nonendothelial sources of PDGF-B regulate pericyte recruitment and influence vascular pattern formation in tumors. J Clin Invest 112: 1142-1151, 2003.

4 Drolet DW, Green LS, Gold L and Janjic N: Fit for the Eye: Aptamers in Ocular Disorders. Nucleic Acid Ther 26: 127-146, 2016. doi:10.1089/nat.2015.0573, 2016.

5 Matei D, Kelich S, Cao L, Menning N, Emerson RE, Rao J, Jeng $\mathrm{MH}$, and Sledge GW: PDGF-BB induces VEGF secretion in ovarian cancer. Cancer Biol Ther 6: 1951-1959, 2007.

6 Lu C, Shahzad MM, Moreno-Smith M, Lin YG, Jennings NB, Allen JK, Landen CN, Mangala LS, Armaiz-Pena GN, Schmandt R, Nick AM, Stone RL, Jaffe RB, Coleman RL and Sood AK: Targeting pericytes with a PDGF-B aptamer in human ovarian carcinoma models. Cancer Biol Ther 9: 176-182, 2010.

7 Choi HJ, Armaiz Pena GN, Pradeep S, Cho MS, Coleman RL and Sood AK: Anti-vascular therapies in ovarian cancer: moving beyond anti-VEGF approaches. Cancer Metastasis Rev 34: 1940, 2015.

8 Hansen JM, Coleman RL and Sood AK: Targeting the tumour microenvironment in ovarian cancer. Eur J Cancer 56: 131-143, 2016.

9 Muggia FM: Intraperitoneal Therapy for Ovarian Cancer. J Clin Oncol 34: 882, 2016.

10 Ruscito I, Gasparri ML, Marchetti C, De Medici C, Bracchi C, Palaia I, Imboden S, Mueller MD, Papadia A, Muzii L and Panici PB: Cediranib in ovarian cancer: state of the art and future perspectives. Tumour Biol 37: 2833-2839, 2016. 
11 Colombo N, Conte PF, Pignata S, Raspagliesi F and Scambia G.Bevacizumab in ovarian cancer: Focus on clinical data and future perspectives. Crit Rev Oncol Hematol 97: 335-348, 2016.

12 Symeonides S and Gourley C: Ovarian Cancer Molecular Stratification and Tumor Heterogeneity: A Necessity and a Challenge. Frontiers in Oncology 5: 229, 2015.

13 Oza AM, Cibula D, Benzaquen AO, Poole C, Mathijssen RH, Sonke GS, Colombo N, Špaček J, Vuylsteke P, Hirte H, Mahner S, Plante M, Schmalfeldt B, Mackay H, Rowbottom J, Lowe ES, Dougherty B, Barrett JC and Friedlander M: Olaparib combined with chemotherapy for recurrent platinum-sensitive ovarian cancer: a randomised phase 2 trial. Lancet Oncol 16: 87-97, 2015 .

14 du Bois A, Floquet A, Kim JW, Rau J, del Campo JM and Friedlander M: Incorporation of pazopanib in maintenance therapy of ovarian cancer. J Clin Oncol 32: 3374-3382, 2014.

15 Matulonis UA, Berlin S, Ivy P, Tyburski K, Krasner C, Zarwan C, Berkenblit A, Campos S, Horowitz N, Cannistra SA, Lee H, Lee J, Roche M, Hill M, Whalen C, Sullivan L, Tran C, Humphreys BD and Penson RT: Cediranib, an oral inhibitor of vascular endothelial growth factor receptor kinases, is an active drug in recurrent epithelial ovarian, fallopian tube, and peritoneal cancer. J Clin Oncol 27: 5601-5606, 2009.

16 Baumann KH, du Bois A, Meier W, Rau J, Wimberger P, Sehouli J, Kurzeder C, Hilpert F, Hasenburg A, Canzler U, Hanker LC, Hillemanns P, Richter B,Wollschlaeger K, Dewitz T, Bauerschlag D and Wagner U: A phase II trial (AGO 2.11) in platinum-resistant ovarian cancer: a randomized multicenter trial with sunitinib (SU11248) to evaluate dosage, schedule, tolerability, toxicity and effectiveness of a multitargeted receptor tyrosine kinase inhibitor monotherapy. Ann Oncol 23: 22652271, 2012.

17 Hainsworth JD, Thompson DS, Bismayer JA, Gian VG, Merritt WM, Whorf RC, Finney LH and Dudley BS: Paclitaxel/carboplatin with or without sorafenib in the first-line treatment of patients with stage III/IV epithelial ovarian cancer: a randomized phase II study of the Sarah Cannon Research Institute. Cancer Med 4: 673-681, 2015.

18 Safra T, Andreopoulou E, Levinson B, Borgato L, Pothuri B, Blank S, Tiersten A, Boyd L, Curtin J and Muggia F: Weekly paclitaxel with intermittent imatinib mesylate (Gleevec): tolerance and activity in recurrent epithelial ovarian cancer. Anticancer Res 30: 3243-3247, 2010.

19 Raica M and Cimpean AM: Platelet-Derived Growth Factor (PDGF)/PDGF Receptors (PDGFR) Axis as Target for Antitumor and Antiangiogenic Therapy. Pharmaceuticals 3: 572-599, 2010.

20 Kacinski BM, Carter D, Kohorn EI, Mittal K, Bloodgood RS, Donahue J, Kramer CA and Fischer D: Oncogene expression in vivo by ovarian adenocarcinomas and mixed-mullerian tumors. The Yale Journal of Biology and Medicine 62: 379-392, 1989.
21 Versnel MA, Haarbrink M, Langerak AW, de Laat PA, Hagemeijer A, van der Kwast TH, van den Berg-Bakker LA and Schrier PI: Human ovarian tumors of epithelial origin express PDGF in vitro and in vivo. Cancer Genet Cytogenet 73: 60-64, 1994.

22 Yamamoto S, Tsuda H, Takano M, Kita T, Kudoh K, Furuya K, Tamai S and Matsubara O: Expression of platelet-derived growth factors and their receptors in ovarian clear-cell carcinoma and its putative precursors. Mod Pathol 21: 115-124, 2008.

23 Song SH, Jeong IG, You D, Hong JH, Hong B, Song C, Jung WY, Cho YM, Ahn H and Kim CS: VEGF/VEGFR2 and PDGF$\mathrm{B} / \mathrm{PDGFR}-\beta$ expression in non-metastatic renal cell carcinoma: a retrospective study in 1,091 consecutive patients. Int J Clin Exp Pathol 7: 7681-7689, 2014.

24 Shim M, Song C, Park S, Choi SK, Cho YM, Kim CS and Ahn $\mathrm{H}$ : Prognostic significance of platelet-derived growth factor receptor- $\beta$ expression in localized clear cell renal cell carcinoma. J Cancer Res Clin Oncol 141: 2213-2220, 2015.

25 Versnel MA, Hagemeijer A, Bouts MJ, van der Kwast TH and Hoogsteden HC: Expression of c-sis (PDGF B-chain) and PDGF A-chain genes in ten human malignant mesothelioma cell lines derived from primary and metastatic tumors. Oncogene 2: 601$605,1988$.

26 Savolainen-Peltonen H, Loubtchenkov M, Petrov L, Delafontaine $\mathrm{P}$ and Häyry P: Estrogen regulates insulin-like growth factor 1, platelet-derived growth factor $\mathrm{A}$ and $\mathrm{B}$, and their receptors in the vascular wall. Transplantation 77: 35-42, 2004.

27 Rizvi S, Mertens JC, Bronk SF, Hirsova P, Dai H, Roberts LR, Kaufmann SH and Gores GJ: Platelet-derived growth factor primes cancer-associated fibroblasts for apoptosis. J Biol Chem 289: 22835-22849, 2014.

28 Pinto MP, Dye WW, Jacobsen BM and Horwitz KB: Malignant stroma increases luminal breast cancer cell proliferation and angiogenesis through platelet-derived growth factor signaling. BMC Cancer 14: 735, 2014.

29 Lederle W, Stark HJ, Skobe M, Fusenig NE and Mueller MM: Platelet-derived growth factor-BB controls epithelial tumor phenotype by differential growth factor regulation in stromal cells. Am J Pathol 169: 1767-1783, 2006.
Received August 4, 2016

Revised September 20, 2016

Accepted September 22, 2016 